\title{
Should Fallopian Tubes Be Removed During Hysterectomy Procedures? - A Statement by AGO Ovar
}

\author{
Sollen die Tuben im Rahmen der Hysterektomie entfernt werden? - \\ Ein Statement der AGO Ovar
}

Authors

Affiliations
M. Pölcher ${ }^{1}$, S. Hauptmann ${ }^{2}$, C. Fotopoulou ${ }^{3}$, B. Schmalfeldt ${ }^{4}$, I. Meinhold-Heerlein ${ }^{5}$, A. Mustea ${ }^{6}$, I. Runnebaum ${ }^{7}$, J. Sehouli ${ }^{8}$, for the Kommission Ovar of the Gynecologic Oncology Study Group (AGO)

The affiliations are listed at the end of the article.
Deutschsprachige Zusatzinformationen online abrufbar unter: www.thieme-connect.de/ ejournals/toc/gebfra

\section{Bibliography}

DOI http://dx.doi.org/

10.1055/s-0035-1545958

Geburtsh Frauenheilk 2015; 75 :

339-341 @ Georg Thieme

Verlag KG Stuttgart . New York. ISSN 0016-5751

\section{Correspondence \\ Professor Dr. med. \\ Jalid Sehouli}

Gynäkologisches Tumorzentrum und Europäisches Kompetenzzentrum für Eierstockkrebs (EKZE)

Campus Virchow Klinikum und Benjamin Franklin Comprehensive Cancer Center (CCCC)

Universitätsmedizin Berlin Charité

Augustenburger Platz 1

13353 Berlin

jalid.sehouli@charite.de

\section{Introduction \\ $\nabla$}

The detection of premalignant cells in the epithelia of the fallopian tubes has revolutionized the theories on the genesis of ovarian cancer. Occurrence of serous tubal intraepithelial carcinomas (STIC) has been established in carriers of the germline mutation of the BRCA1/2 gene, with STICs now considered as a possible origin of serous high-grade carcinomas which frequently occur in this patient population. Bilateral salpingooophorectomy leads to a significant decrease in the risk of developing this carcinoma. It is recommended that this prophylactic surgery be performed between the age of 40 and 45 years.

Removal of the fallopian tubes has been propagated analogously in women with no proven genetic or identifiable familial risk of ovarian cancer. Fallopian tube removal has been proposed during surgery for benign disease, for example in women in whom hysterectomy is indicated. Such "prophylactic" or "opportunistic" salpingectomy procedures could prevent carcinoma development in later years. Critics warn that such an intervention could result in inadequate blood supply to the ovaries, resulting in morbidity associated with early hormone deficiency. It is still unclear how effective opportunistic salpingectomy could be as a primary prevention strategy and how such a strategy should best be implemented.

\section{Are All Serous Ovarian Cancers Tubal Carcinomas? \\ $\nabla$}

The detection of a group of in-situ lesions in the tubal fimbria [1] abruptly shifted the scientific focus to the fallopian tubes; most putative ovarian cancers could have a tubal origin with spread to the ovarian cortex occurring through drainage to the ovarian surface or through displacement of transformed tubal epithelium during ovulation and thus only appear to originate in the ovary. Histopathologically, this tubal cancerogenesis must be understood as a stepwise process, starting with p53 signature and moving from proliferative p53 signature to serous intraepithelial carcinoma (STIC) [2-13]. Intensive workup of the distal fallopian tubes demonstrated the following incidence for STIC:

- in $2-7 \%$ of cases undergoing risk-reduction salpingo-oophorectomy (BRCA1/2-positive women or women with a familial risk) [14],

- in up to $80 \%$ of surgical specimens of BRCA1/2 patients with serous high-grade carcinoma, and

- in $46 \%$ of cases with sporadic ovarian cancer $[12,15]$.

Development from a tubal precursor cannot be proven in $40-80 \%$ of cases if the patient with ovarian cancer has no underlying BRCA 1 or 2 mutation. A causal relationship is likely when STIC occurs coincidentally with serous high-grade carcinoma, but parallel independent occurrence as a result of field cancerogenesis is also conceivable. It is therefore unlikely that all serous ovarian cancers originate from tubal epithelia; rather, it is likely that there are several different pathways for the development of serous high-grade cancers. Prophylactic tubal removal will not prevent the development of all cancers in later years.

\section{Advantages and Disadvantages of Prophylactic Adnexal Surgery}

The removal of both adnexae during hysterectomy procedures to prevent the development of ovarian cancer has reduced the incidence in prospective cohort studies but did not reduce mortality $[16,17]$. Premenopausal women develop a higher risk of osteoporosis and cardiovascular dis- 
ease, which appears statistically to compensate for the prophylactic effect on carcinogenesis [18].

Ovarian function is assumed to play an important role on sexual function and quality-of-life even after menopause, so that there are no general recommendations whether and from what age the adnexae should be removed during hysterectomy.

Tubal removal during hysterectomy can affect blood supply to the ovaries and lead to detrimental degenerative effects and earlier menopause [19]. A number of studies using various methods and end-points such as Doppler measurement of ovarian blood flow or serial hormonal measurements (FSH, LH, estradiol, antiMüllerian hormone) showed no effect on ovarian reserve [2023]. But because of the relatively small patient populations investigated in these studies, it is not possible to come to a conclusive evalution. There are no controlled studies on the potential early start of menopause after salpingectomy. Perioperative morbidity after hysterectomy with bilateral salpingectomy was not increased compared to hysterectomy without salpingectomy [24]. Conversely, hysterectomy with retention of fallopian tubes or sterilization is associated with more adverse effects compared to the potential disadvantages of hysterectomy procedures with salpingectomy. Subsequent hydrosalpinx can require surgical investigation. Retention of fallopian tubes doubles the risk for later surgery of adnexae, indicating a benefit of tubal removal $[24,25]$.

\section{How Effective Could Removal of the Fallopian Tubes Be? $\nabla$}

Around 125,000 hysterectomies are performed annually in Germany [26]. If salpingectomy performed during hysterectomy procedures is the only measure taken to reduce the incidence of ovarian cancer, a simple calculation puts the hopes of a significant impact into perspective: out of 8000 new cases with disease (including those of genetic origin) round $70 \%$ are serous highgrade carcinomas. If opportunistic salpingectomy were to be carried out immediately in all hysterectomy procedures and would thereby prevent the development of all serous cancers, the incidence of ovarian cancer would be reduced by around $2.3 \%$ in 20 years - if all hysterectomies were carried out together with bilateral salpingectomies and thus all serous cancers could be prevented. If - as is assumed - other mechanisms can also lead to the development of serous high-grade cancers even after bilateral salpingectomy, the effect will be lower. It is not clear to what extent tubal removal has a protective effect against the development of endometrioid or clear-cell ovarian cancers. Epidemiological data have shown an overall lower risk of developing ovarian cancer after tubal sterilization. It is possible that carcinogenic substances are transported through the tubes; opportunistic salpingectomy could thus reduce the incidence of non-serous subtypes.

\section{How Could Opportunistic Salpingectomy be Implemented as a Primary Prevention Strategy? $\nabla$}

If prophylactic surgical tubal removal is considered as a preventive strategy, the greatest reduction in the incidence of ovarian cancer in a low-risk population could be achieved if tubal removal is carried out rigorously and frequently. The presumably simplest and currently most common form of opportunistic salpingectomy is carried out during abdominal or laparoscopic hysterectomies where surgical access provides a good opportunity to carry out ovarian and tubal procedures. But should a general recommendation for salpingectomy then also apply to vaginal hysterectomy procedures in which salpingectomy is technically not always easy? Should the recommendation be expanded to include other gynecological procedures such as surgery for ovarian cysts? Should every woman be offered bilateral salpingectomy after she has completed her family if she is scheduled for intraabdominal surgery, e.g., cholecystectomy? Will this result in a modification of the standard technique for tubal sterilization with the procedure changed to bilateral salpingectomy for preventive reasons? Could failure to educate a patient about tubal removal have legal ramifications if a patient later develops serous high-grade cancer and she was not informed about the potential association prior to undergoing hysterectomy? Answers to these questions and any conclusive assessment of the effects of opportunistic salpingectomy procedures will probably only be possible in several years' time.

\section{Conclusion \\ $\nabla$}

In practice, the evidence of precancerous lesions in the fallopian tubes has resulted in diagnoses for opportunistic salpingectomy during hysterectomy being given with a liberal hand. There are no indications that opportunistic salpingectomy is associated with increased morbidity; the rates for surgical interventions required at a later date appear to be much lower in women who underwent opportunistic salpingectomy than for women with retained fallopian tubes. Based on the current scientific data it is not possible to give a general recommendation in support of opportunistic salpingectomy for cancer prevention. Prospective registries and long-term data collection will be necessary prior to making such a recommendation. During patient consultations and discussions prior to undergoing surgery, the patient must be informed about the possibility of tubal removal and the potential risks and benefits associated with bilateral salpingectomy. A detailed description of the current scientific position on opportunistic salpingectomy has been published in Archives of Gynecology and Obstetrics [27].

\section{Affiliations}

${ }^{1}$ Frauenklinik Taxisstraße, Rotkreuzklinikum München, Munich

${ }^{2}$ Institut für Pathologie, Krankenhaus Düren, Düren

3 West London Gynaecological Cancer Centre and Ovarian Cancer Action Research Centre Imperial College Healthcare Trust London, London

${ }^{4}$ Frauenklinik des Klinikums rechts der Isar der Technischen Universität München, Munich

${ }^{5}$ Klinik für Gynäkologie und Geburtsmedizin, Universitätsklinikum RWTH Aachen, Aachen

${ }^{6}$ Klinik und Poliklinik für Frauenheilkunde und Geburtshilfe, Universitätsmedizin Greifswald, Greifswald

${ }^{7}$ Klinik für Frauenheilkunde und Geburtshilfe, Universitätsklinikum Jena, Jena

${ }^{8}$ Benjamin Franklin Charité Comprehensive Cancer Center (CCCC) Charité/ Universitätsmedizin Berlin Charité, Berlin

\section{References}

1 Crum CP, Drapkin R, Miron A et al. The distal Fallopian tube: a new model for pelvic serous carcinogenesis. Curr Opin Obstet Gynecol 2007; 19: 3-9

2 Piek JM, van Diest PJ, Zweemer RP et al. Dysplastic changes in prophylactically removed Fallopian tubes of women predisposed to developing ovarian cancer. J Pathol 2001; 195: 451-456

3 Medeiros F, Muto MG, Lee Yet al. The tubal fimbria is a preferred site for early adenocarcinoma in women with familial ovarian cancer syndrome. Am J Surg Pathol 2006; 30: 230-236 
4 Kindelberger DW, Lee Y, Miron A et al. Intraepithelial carcinoma of the fimbria and pelvic serous carcinoma: Evidence for a causal relationship. Am J Surg Pathol 2007; 31: 161-169

5 Folkins AK, Jarboe EA, Roh MH et al. Precursors to pelvic serous carcinoma and their clinical implications. Gynecol Oncol 2009; 113: 391-396

6 Powell CB, Chen LM, McLennan J et al. Risk-reducing salpingo-oophorectomy (RRSO) in BRCA mutation carriers: experience with a consecutive series of 111 patients using a standardized surgical-pathological protocol. Int J Gynecol Cancer 2011; 21: 846-851

7 Chivukula M, Neimeier LA, Edwards R et al. Carcinomas of distal Fallopian tube and their association with tubal intraepithelial carcinoma: do they share a common "precursor" lesion? Loss of heterozygosity and immunohistochemical analysis using PAX 2, WT-1, and P53 markers. ISRN Obstet Gynecol 2011; 2011: 858647

8 Paik DY, Janzen DM, Schafenacker AM et al. Stem-like epithelial cells are concentrated in the distal end of the fallopian tube: a site for injury and serous cancer initiation. Stem Cells 2012; 30: 2487-2497

9 Tang S, Onuma K, Deb P et al. Frequency of serous tubal intraepithelial carcinoma in various gynecologic malignancies: a study of 300 consecutive cases. Int J Gynecol Pathol 2012; 31: 103-110

10 Bijron JG, Seldenrijik CA, Zweemer RP et al. Fallopian tube intraluminal tumor spread from noninvasive precursor lesions: a novel metastatic route in early pelvic carcinogenesis. Am J Surg Pathol 2013; 37: 11231130

11 Gao FF, Bhargava R, Yang H et al. Clinicopathologic study of serous tubal intraepithelial carcinoma with invasive carcinoma: is serous tubal intraepithelial carcinoma a reliable feature for determining the organ of origin? Hum Pathol 2013; 44: 1534-1543

12 Lee S, Nelson G, Duan Q et al. Precursor lesions and prognostic factors in primary peritoneal serous carcinoma. Int J Gynecol Pathol 2013; 32: 547-555

13 Reitsma W, de Bock GH, Oosterwijk JC et al. Support of the 'Fallopian tube hypothesis' in a prospective series of risk-reducing salpingooophorectomy specimens. Eur J Cancer 2013; 49: 132-141

14 Callahan MJ, Crum CP, Medeiros F et al. Primary fallopian tube malignancies in BRCA-positive women undergoing surgery for ovarian cancer risk reduction. J Clin Oncol 2007; 25: 3985-3990

15 Przybycin CG, Kurman RJ, Ronnett BM et al. Are all pelvic (nonuterine) serous carcinomas of tubal origin? Am J Surg Pathol 2010; 34: 14071416
16 Jacoby VL, Grady D, Wactawski-Wende J et al. Oophorectomy vs. ovarian conservation with hysterectomy: cardiovascular disease, hip fracture, and cancer in the Women's Health Initiative Observational Study. Arch Intern Med 2011; 171: 760-768

17 Parker WH, Feskanich D, Broder MS et al. Long-term mortality associated with oophorectomy compared with ovarian conservation in the nurses' health study. Obstet Gynecol 2013; 121: 709-716

18 Rocca WA, Grosshardt BR, de Andrade $M$ et al. Survival patterns after oophorectomy in premenopausal women: a population-based cohort study. Lancet Oncol 2006; 7: 821-828

19 Repasy I, Lendvai V, Koppan A et al. Effect of the removal of the Fallopian tube during hysterectomy on ovarian survival: the orphan ovary syndrome. Eur J Obstet Gynecol Reprod Biol 2009; 144: 64-67

20 Sezik M, Ozkaya O, Demir F et al. Total salpingectomy during abdominal hysterectomy: effects on ovarian reserve and ovarian stromal blood flow. J Obstet Gynaecol Res 2007; 33: 863-869

21 Petri Nahás EA, Pontes A, Nahas-Neto J et al. Effect of total abdominal hysterectomy on ovarian blood supply in women of reproductive age. J Ultrasound Med 2005; 24: 169-174

22 Findley $A D$, Siedhoff $M T$, Hobbs KA et al. Short-term effects of salpingectomy during laparoscopic hysterectomy on ovarian reserve: a pilot randomized controlled trial. Fertil Steril 2013; 100: 1704-1708

23 Dar P, Sachs GS, Strassburger D et al. Ovarian function before and after salpingectomy in artificial reproductive technology patients. Hum Reprod 2000; 15: 142-144

24 Vorwergk J, Radosa MP, Nicolaus $K$ et al. Prophylactic bilateral salpingectomy (PBS) to reduce ovarian cancer risk incorporated in standard premenopausal hysterectomy: complications and re-operation rate. J Cancer Res Clin Oncol 2014; 140: 859-865

25 Guldberg R, Wehberg S, Wessel Skovlund C et al. Salpingectomy as standard at hysterectomy? A Danish cohort study, 1977-2010. BMJ Open 2013; 3: e002845

26 Müller A, Thiel FC, Renner SP et al. Hysterectomy-a comparison of approaches. Dtsch Arztebl Int 2010; 107: 353-359

27 Pölcher M, Hauptmann S, Fotopoulou CH et al. Opportunistic salpingectomies for the prevention of a high-grade serous carcinoma: a statement by the Kommission Ovar of the AGO. Arch Gynecol Obstet 2015; DOI: $10.1007 / \mathrm{s} 00404-015-3697-y$ 